\title{
Lesion size and circumferential range identified as independent risk factors for esophageal stricture after endoscopic submucosal dissection
}

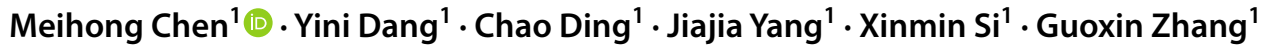

Received: 1 August 2019 / Accepted: 4 January 2020 / Published online: 17 January 2020

(c) The Author(s) 2020

\begin{abstract}
Background and aim Endoscopic submucosal dissection (ESD) is used to treat early esophageal cancer and precancerous lesions. Patients undergoing ESD are prone to esophageal stenosis, which impairs therapeutic efficacy and quality of life. This retrospective study aimed to investigate the potential association between patient demographics and esophageal lesion characteristics with the risk of esophageal stenosis following ESD.

Methods For this retrospective study 190 consecutive patients who underwent ESD between January 2013 and January 2015 were recruited. Data on patient demographics, esophageal lesion-related factors, operation details, esophageal stenosis occurrence and measures taken to prevent or treat stricture were collected, and the normality of distribution of each indicator was assessed with a Kolmogorov-Smirnov test. Stenosis risk factors were then identified using univariate and multivariate logistic regression.

Results Post-ESD esophageal stenosis occurred in 51 cases. Multivariate logistic regression analysis was performed to identify independent risk factors. A history of EMR/ESD $(\mathrm{OR}=4.185,95 \% \mathrm{CI}: 1.511-11.589)$, resection circumferential diameter $(\mathrm{OR}=1.721,95 \% \mathrm{CI}: 1.135-2.610)$, non-en bloc resection ( $\mathrm{OR}=7.413,95 \% \mathrm{CI}: 2.398-22.921)$, submucosal infiltration $(\mathrm{OR}=3.449,95 \% \mathrm{CI}: 1.014-11.734)$ and circumferential resection range $(\mathrm{OR}=57.493,95 \% \mathrm{CI}: 17.236-191.782)$ were identified as independent risk factors for post-ESD esophageal stenosis. Spraying porcine fibrin adhesive on the resection bed reduced neither the incidence of postoperative stenosis nor the extent of postoperative dilation.

Conclusion Post-ESD esophageal stenosis is significantly related to size and circumferential range of lesion resection. EMR/ ESD history, non-en bloc resection and submucosal infiltration may be additional risk factors.
\end{abstract}

Keywords Endoscopic submucosal dissection (ESD) $\cdot$ Esophageal stenosis $\cdot$ Risk factors $\cdot$ Retrospective

Early esophageal cancer and precancerous lesions are confined to the submucosa, with low risk of distant metastasis. Endoscopic resection has become the first-line therapy for the treatment of early esophageal neoplasia. Unlike endoscopic mucosal resection (EMR), endoscopic submucosal

Meihong Chen and Yini Dang have contributed equally to this article.

Guoxin Zhang

guoxinz@njmu.edu.cn

1 Department of Gastroenterology, Jiangsu Province Hospital and The First Affiliated Hospital of Nanjing Medical University, First Clinical Medical College of Nanjing Medical University, 300 Guangzhou road, Gulou district, Nanjing, Jiangsu, China dissection (ESD) allows en bloc resection regardless of lesion size, reducing the risk of recurrence and facilitating precise histologic staging. Therefore, ESD may be superior to EMR in the treatment of early esophageal cancer, due to significantly higher en bloc and curative resection rates and lower local recurrence rates [1,2]. Furthermore, ESD can effectively remove lesions, leading to less trauma, fewer complications, no reported mortality, and higher quality of life for patients. The long-term efficacy of ESD is comparable to that of surgery, so ESD has become the standard therapeutic technique for early esophageal cancer [3, 4]. However, it is noteworthy that acute inflammatory reaction and fibrous connective tissue hyperplasia following extensive endoscopic resection can lead to esophageal stenosis, changes in eating behavior, and aspiration pneumonia in patients [5]. There is currently no consensus regarding the 
relationship between ESD and the development of postoperative esophageal stricture.

\section{Methods}

\section{Patient selection}

Relevant information on patients undergoing esophageal ESD between January 2013 and January 2015 was provided by the electronic medical records of the Jiangsu Provincial People's Hospital. A total of 229 patients underwent esophageal ESD, regardless of the pre- or postoperative pathology. In China, the incidence of esophageal squamous cell carcinoma (including high/low grade intraepithelial neoplasia) is considerably higher than that of adenocarcinoma and Barrett's esophagus. In Europe and the United States, the predominant histologic subtype is adenocarcinoma [6]. In order to reduce heterogeneity of the patient population, patients with Barrett's esophagus or adenocarcinoma were excluded. The inclusion criteria for the study were as follows: (1) The pathological diagnosis was low grade intraepithelial neoplasia (LGIN), high grade intraepithelial neoplasia (HGIN), or squamous cell carcinoma; (2) No additional surgery or chemoradiotherapy was performed after ESD.

This study has been approved by the Ethics Committee of the Jiangsu Provincial People's Hospital and written informed consent was obtained from each patient. The ethical approval number for this study was 2018-SR-213.

\section{Data collection}

All patients had a follow-up exam within six months of undergoing ESD. The outcome of interest at follow-up was the presence of esophageal stenosis. The enrolled patients were divided into stenosis and non-stenosis groups based on the following definition of esophageal stenosis: narrowing of the esophageal lumen to the extent that it becomes impassable by an ordinary endoscope (diameter $9.8 \mathrm{~mm}$ ) and an accompanying difficulty in consuming solid foods [7]. Demographic information including age, sex, body mass index (BMI), smoking history, drinking history, comorbidity and family history of esophageal cancer was collected. Lesion characteristics including pre- and postoperative pathology, location, lesion number, ultrasonic infiltration, morphology, longitudinal resection length, circumferential specimen size, circumferential range and depth of infiltration were recorded. Procedural details including 'lifting sign', electric coagulation, en bloc resection, muscular injury, perforation, hemorrhage, clip number, and operating time were also collected.

\section{Statistical analysis}

Statistical analysis was performed using the SPSS Statistics 20.0 program (SPSS, Chicago, Illinois, USA). Due to the large number of potential risk factors, we first screened the variables with univariate analysis and identified those that appeared to differ between the stenosis and non-stenosis groups $(P<0.05)$. Firstly, we used the Kolmogorov-Smirnov test to analyze the normality of the distribution of each indicator. For normally distributed continuous variables, a Student's $t$-test was then conducted to assess the intergroup differences and results were reported as mean \pm SD. The Wilcoxon rank-sum test was used for continuous non-normal data. For discrete data, the Fisher's exact and chi-squared tests were performed in order to analyze differences between proportions in the stenosis and non-stenosis groups.

We then conducted multivariate logistic regression analyses with the significant variables in order to evaluate the association between esophageal stricture and potential risk factors.

\section{Results}

A total of 190 patients, with 222 lesions, fulfilled the inclusion criteria and entered the analyses. There were 51 patients $(26.8 \%)$ who developed stenosis by the time of follow-up, whereas no stenosis was present in 139 patients $(73.2 \%)$ at follow-up. Patients were divided into two groups for analyses, namely a stenosis group and a non-stenosis group. Demographic information, lesion characteristics and procedural details for each of the two groups are depicted in Tables 1-5.

\section{Univariate analysis of demographic information}

The mean age for the non-stenosis group was similar to that of the stenosis group $(63.4 \pm 7.5$ years vs. $64.8 \pm 8.1$ years, $P=0.278$ ). The number of male patients was twice the number of females in each group. Univariate analyses highlighted that a history of gastrointestinal EMR/ESD (17 vs. $15, P=0.005)$ was significantly different between the two groups; patients in the non-stenosis group were less likely to have a history of gastrointestinal EMR/ESD compared to those in the stenosis group. However, there were no significant differences found between the two groups in lesion number, age, sex, BMI, smoking history, drinking history, hypertension, diabetes, coronary heart disease, stroke, chronic obstructive pulmonary disease (COPD), gallbladder surgery, other cancers or family history (Table 1). 
Table 1 Univariate analysis of patient characteristics

\begin{tabular}{llll}
\hline Patient characteristics & Non-stenosis & Stenosis & $P$ value \\
\hline Number, $n$ & 139 & 51 & \\
Lesion number, $n$ & 160 & 62 & 0.889 \\
Age, mean \pm SD, year & $63.4 \pm 7.5$ & $64.8 \pm 8.1$ & 0.278 \\
Sex, male/female, $n$ & $100 / 39$ & $32 / 19$ & 0.222 \\
BMI, mean \pm SD & $23.2 \pm 3.1$ & $23.3 \pm 2.9$ & 0.882 \\
Smoking history, $n$ & 54 & 13 & 0.088 \\
Drinking history, $n$ & 42 & 14 & 0.711 \\
Comorbidity, $n$ & & & \\
Hypertension & 38 & 17 & 0.419 \\
Diabetes & 8 & 1 & 0.48 \\
Coronary heart disease & 5 & 2 & 1 \\
Stroke & 5 & 5 & 0.183 \\
COPD & 4 & 1 & 1 \\
Gallbladder surgery & 9 & 0 & 0.14 \\
Gastrointestinal EMR/ESD & 17 & 15 & $0.005^{*}$ \\
Other cancers & 11 & 4 & 1 \\
Family history, $n$ & 22 & 8 & 0.981 \\
\hline
\end{tabular}

$B M I$ Body Mass Index, $C O P D$ chronic obstructive pulmonary disease *Indicates that the results were statistically significant

\section{Univariate analysis of lesion characteristics}

There were no significant differences between the two groups regarding the lesion location, lesion number, degree of ultrasonic infiltration, or morphology $(P>0.05)$. However, there were significant differences found between the two groups regarding preoperative pathology, longitudinal resection length, circumferential specimen size, circumferential range, postoperative pathology and depth of infiltration $(P<0.05)$ (Table 2$)$. We performed further subgroup analysis based on circumferential range, as preoperative pathology had not been mentioned in previous studies as a possible risk factor for the development of esophageal stenosis. However, we did not identify any conspicuous differences in preoperative pathology between each subgroup (Table 3).

\section{Univariate analysis of factors related to ESD procedure}

Specific factors related to the ESD procedure may influence the risk of developing postoperative esophageal stenosis. Univariate analysis of procedure characteristics indicated that lift sign, repeated electric coagulation, en bloc resection, muscular injury, perforation and operation time were associated with the presence of stenosis at follow-up $(P<0.05)$, whereas hemorrhage and number of clips were not $(P>0.05)$ (Table 4).
Results from the univariate analyses therefore suggest that the following factors are associated with the development of postoperative esophageal ESD stenosis: a history of gastrointestinal EMR/ESD, preoperative pathology, longitudinal resection length, maximum specimen size, circumferential range, postoperative pathology, depth of infiltration, lift sign, repeated electric coagulation, en bloc resection, muscular injury, perforation and operation time.

\section{Multivariate logistic regression analyses}

The above indicators that were identified as being significantly different between the stenosis and non-stenosis group through univariate analyses were then included in multivariate analyses for further verification. Logistic regression analyses determined that a history of gastrointestinal EMR/ ESD $(\mathrm{OR}=4.185,95 \% \mathrm{CI}: 1.511-11.589)$, circumferential specimen size (OR $=1.721,95 \% \mathrm{CI}$ : $1.135-2.610)$, circumferential range $(\mathrm{OR}=57.493,95 \%$ CI: $17.236-191.782)$, depth of infiltration (OR $=3.449,95 \%$ CI: $1.014-11.734)$ and nonen bloc resection ( $\mathrm{OR}=7.413,95 \%$ CI: $2.398-22.921)$ were independent risk factors for postoperative stenosis at follow-up (Table 5).

\section{Prevention and treatment of esophageal stricture}

All patients admitted to the study were routinely treated with proton pump inhibitors (PPI) therapy and mucosal protectants after ESD. In addition, a total of 34 patients in our retrospective study were treated with a porcine fibrin adhesive during ESD. Eleven of them developed esophageal stenosis. It seemed that using porcine fibrin adhesive was not associated with lower incidence of stenosis $(\mathrm{OR}=1.387,95 \% \mathrm{CI}$ : 0.621-3.097), nor the mean number of stenosis treatments $(3.1 \pm 4.2$ vs $4.2 \pm 5.3, P=0.480)$. No patients included in this study received intraoperative prophylactic local steroid injections. Patients who took oral steroids were not found to have a lower incidence of stenosis, or lower number of dilations, compared to those who did not take steroids $(3.1 \pm 4.2$ vs $7 \pm 7.9$, $P=0.142$ ), so it does not make sense now.

A total of 43 patients in the stenosis group received poststenosis treatment. The treatment methods included Bougie expansion, stent implantation, expansion combined with stent implantation and expansion combined with drug injection. We found that different treatments were associated with different outcomes, but we could not determine which was the best treatment (Table 6). 
Table 2 Univariate analysis of lesion characteristics

\begin{tabular}{|c|c|c|c|}
\hline Lesion characteristics & Non-stenosis & Stenosis & $P$ value \\
\hline Preoperative pathology, $n$ & & & $0.001 *$ \\
\hline LGIN & 56 & 8 & \\
\hline HGIN & 83 & 43 & \\
\hline Location, $n$ & & & 0.698 \\
\hline Upper thoracic part & 6 & 3 & \\
\hline Middle thoracic part & 78 & 31 & \\
\hline Lower thoracic part & 55 & 17 & \\
\hline Lesion number, $n$ & & & 0.103 \\
\hline Single & 120 & 39 & \\
\hline Multiple & 19 & 12 & \\
\hline Ultrasonic infiltration, $n$ & & & 0.357 \\
\hline Mucosal & 97 & 32 & \\
\hline Submucosal & 42 & 19 & \\
\hline Morphology & & & 0.774 \\
\hline Flat & 129 & 46 & \\
\hline Protruded & 10 & 5 & \\
\hline Depressed & 0 & 0 & \\
\hline Longitudinal resection length, medium $(\mathrm{cm})$ & 6 & 7 & $<0.001 *$ \\
\hline Circumferential specimen size, medium, $(\mathrm{cm})$ & 3 & 4 & $<0.001 *$ \\
\hline Circumferential range, $n$ & & & $<0.001^{*}$ \\
\hline$<1 / 2$ & 71 & 1 & \\
\hline $1 / 2-3 / 4$ & 65 & 9 & \\
\hline $3 / 4-1$ & 3 & 41 & \\
\hline Postoperative pathology, $n$ & & & $<0.001^{*}$ \\
\hline LGIN & 30 & 0 & \\
\hline HGIN & 94 & 36 & \\
\hline Tis & 14 & 10 & \\
\hline Squamous carcinoma & 1 & 5 & \\
\hline Depth of infiltration, $n$ & & & $<0.001^{*}$ \\
\hline Mucosal & 131 & 39 & \\
\hline Submucosal & 8 & 12 & \\
\hline
\end{tabular}

LGIN low grade intraepithelial neoplasia, HGIN high grade intraepithelial neoplasia, Tis refers to carcinoma in situ

*Indicates that the results were statistically significant

\section{Discussion}

Although ESD has become the leading treatment for early esophageal lesions, the occurrence of postoperative stricture has a significant influence on overall prognosis [8]. Generally, esophageal stricture occurs within 3 weeks after ESD, and the 6-month follow-up is sufficient for the observation of patients. The stenosis rate of this retrospective study was $26.8 \%$ (51/190), as shown in Table 7; our classification of stenosis was based on the definition used in relevant literature [7]. A total of 8 patients in the stenosis group did not receive stenosis treatment after surgery, and were found to have mild esophageal stenosis at follow-up. If we exclude the eight untreated patients, the incidence of stenosis in this retrospective study is only $22.6 \%$. Factors contributing to the relatively high rate of stenosis in our retrospective study may include the relatively small sample size compared to that of other studies [9] and the presence of particularly large lesions in 44 patients in our research. Previous literature suggests that the incidence of stenosis is between $80-100 \%$ in patients with a circumferential mucosal defect of more than three-quarters [8-15]. Therefore, it is plausible that our study found a higher incidence of total esophageal stricture due to the high percentage of patients presenting with wide esophageal lesions.

Overall, the results of this retrospective study indicate a number of independent risk factors for the development of esophageal stenosis following ESD, which are in line with findings of other studies [13-21]. Mizuta et al. [13] highlighted that the primary contributor to esophageal stenosis 
Table 3 Subgroup analysis of preoperative pathology

\begin{tabular}{lccc}
\hline & Non-stenosis & Stenosis & $P$ value \\
\hline$<1 / 2$ & & & \\
Preoperative pathology, $n$ & & 0.408 \\
LGIN & 29 & 1 & \\
HGIN & 42 & & \\
$1 / 2-3 / 4$ & & & 0.701 \\
Preoperative pathology, $n$ & & 3 & \\
LGIN & 26 & 6 & \\
HGIN & 39 & & \\
$3 / 4-1$ & & & 0.303 \\
Preoperative pathology, $n$ & & 5 & \\
LGIN & 1 & 36 & \\
HGIN & 2 & & \\
\hline
\end{tabular}

LGIN low grade intraepithelial neoplasia, HGIN high grade intraepithelial neoplasia

Table 4 Univariate analysis of procedure characteristics

\begin{tabular}{|c|c|c|c|}
\hline Procedure characteristics & Non-stenosis & Stenosis & $P$ value \\
\hline Lift sign, $n$ & & & $0.017 *$ \\
\hline Positive & 136 & 45 & \\
\hline Negative & 3 & 6 & \\
\hline Rich blood vessels, $n$ & & & $0.033 *$ \\
\hline Rich & 106 & 46 & \\
\hline Not rich & 33 & 5 & \\
\hline En bloc resection, $n$ & & & $<0.001 *$ \\
\hline Yes & 130 & 32 & \\
\hline No & 9 & 19 & \\
\hline Muscular injury, $n$ & & & $0.005 *$ \\
\hline Yes & 21 & 17 & \\
\hline No & 118 & 34 & \\
\hline Perforation, $n$ & & & $<0.001 *$ \\
\hline Yes & 0 & 6 & \\
\hline No & 139 & 45 & \\
\hline Hemorrhage, $n$ & & & 0.104 \\
\hline Yes & 1 & 3 & \\
\hline No & 128 & 48 & \\
\hline Clips number & 2 & 3 & 0.81 \\
\hline Operating time, medium, min & 60 & 90 & $<0.001 *$ \\
\hline Experience of operator, $n$ & & & 0.971 \\
\hline Average & 13 & 4 & \\
\hline Advanced & 126 & 47 & \\
\hline
\end{tabular}

*Indicates that the results were statistically significant

following ESD was the area of periesophageal mucosal defect exceeding $71 \%$. This study also suggested that circumferential range $(\mathrm{OR}=57.493,95 \% \mathrm{CI}$ : $17.236-191.782)$ and mucosal defect circumference length $(\mathrm{OR}=1.721,95 \%$ CI: 1.135-2.610) are independent risk factors for esophageal
Table 5 Multivariate analysis

\begin{tabular}{lrcr}
\hline Risk factors & \multicolumn{1}{c}{ OR } & \multicolumn{1}{l}{$95 \%$ CI } & $P$ value \\
\hline Pre-ESD history & 4.185 & $1.511-11.589$ & 0.006 \\
Maximum specimen size & 1.721 & $1.135-2.610$ & 0.011 \\
Circumferential range & 57.493 & $17.236-191.782$ & $<0.001$ \\
Depth of infiltration & 3.449 & $1.014-11.734$ & 0.048 \\
Non-en bloc resection & 7.413 & $2.398-22.921$ & 0.001 \\
\hline
\end{tabular}

$O R$ odds ratio, $C I$ confidence interval

Table 6 Treatment received for esophageal stenosis

\begin{tabular}{lrcc}
\hline Measures & $N$ & $\begin{array}{l}\text { The number of } \\
\text { treatments }\end{array}$ & $P$ value \\
\hline Expansion & 21 & $2.1 \pm 1.5$ & \\
Stent implantation & 2 & $1 \pm 0.01$ & \\
Expansion + stent & 17 & $6.5 \pm 6.3$ & \\
Expansion + injection & 3 & $4.3 \pm 2.3$ & \\
Total & 43 & $4.0 \pm 4.6$ & $0.02 *$ \\
\hline
\end{tabular}

$N$ the number of patients

*Indicates that the results were statistically significant

Table 7 Rate of esophageal stenosis for the retrospective study

\begin{tabular}{llll}
\hline & Cases of stenosis & $\begin{array}{l}\text { Cases of non- } \\
\text { stenosis }\end{array}$ & $\begin{array}{l}\text { Prevalence } \\
\text { of stenosis } \\
(\%)\end{array}$ \\
\hline$<3 / 4$ & 10 & 136 & 6.8 \\
$>3 / 4$ & 41 & 3 & 93.2 \\
Total & 51 & 139 & 26.8 \\
\hline
\end{tabular}

stricture. We found that the incidence of stenosis was $70.1 \%$ in patients with mucosal defect of more than $2 / 3$ of the circumferential range. Our retrospective study also suggests that the depth of infiltration $(\mathrm{OR}=3.449,95 \% \mathrm{CI}$ : 1.014-11.734) might be a reliable independent predictor of postoperative stricture, which is consistent with the conclusions of Ono et al. [15].

This study also suggests that patients with a history of gastrointestinal ESD or EMR (OR $=4.185,95 \%$ CI: 1.511-11.589) were more likely to have developed stenosis at follow-up; however, the mechanism underlying this association is unclear and requires further exploration. It is worth noting that we did not conduct an in-depth analysis of patients with a history of ESD treatment; further analyses should include the stratification of patients according to whether they underwent an esophageal, gastric, or intestinal ESD procedure. Additionally, non-en bloc resection (OR $=7.413,95 \%$ CI: 2.398-22.921) was considered an independent risk factor. Segmental excision has many 
potential risks; for example, the risk of tumor recurrence is much higher with fractional resection than with en bloc resection [1,2]. It is difficult to manage resection targets endoscopically and achieve precise resection of tumors and mucosa $[22,23]$. Therefore, we recommend that endoscopists aim to achieve en bloc resection when performing ESD.

Current recommendations suggest that esophageal stenosis is treated with conventional therapies such as endoscopic balloon dilation [24], stents [25] and glucocorticoid administration [26]. Novel strategies such as stem cell therapy [27], autologous epithelial cell membrane repair [28] and gastric mucosal transplantation [29] have recently emerged; however, none of these methods can completely prevent or alleviate esophageal stenosis. This current study suggests that the main function of porcine fibrin adhesive is for hemostasis, wound sealing, the promotion of healing and the prevention of adhesion during ESD, rather than for preventing stenosis. The preventive effect of oral steroids suggested in other studies [30, 31] was not apparent in our study. In addition, our results are statistically limited due to the inconsistent number of patients in each treatment group. Due to the uneven distribution of study population among groups, and the limited data available, it is difficult to make pairwise comparisons. It is therefore not possible to identify the most effective postoperative treatment.

A history of gastrointestinal ESD should be considered a risk factor for the development of esophageal stenosis, and endoscopists should aim to achieve en bloc resection when possible. We suggest a re-evaluation of the indications for esophageal ESD, especially with regards to lesions with wide range and depth. Moreover, our study highlights the importance of a high level of expertise amongst endoscopic surgeons carrying out ESD, to ensure precision, accuracy in locating lesions, and the complete resection of lesions. In a nutshell, this study identified relative risk factors contributing to stenosis formation, and provides suggestions for improving postoperative recovery. In the future, it will provide a theoretical foundation for the prevention and treatment of postoperative esophageal stenosis.

\section{Strengths and limitations}

This retrospective study takes a wide range of indicators, regarding the general condition of the patient, lesion characteristics and ESD operational details, into consideration in analyses in order to comprehensively assess a wide range of possible risk factors associated with postoperative stricture formation. It builds upon previous studies by taking more potential risk factors into consideration. It also considers several risk factors that have not been previously explored in analyses, such as the possible association between a history of gastrointestinal EMR/ESD and stenosis following esophageal ESD. Additionally, we compared the methods of prevention and treatment of stenosis for patients in this study.

Retrospective studies are susceptible to selection bias and recall bias, and the definition of symptoms or diseases may change over time, as was the case in this study with the definition of esophageal stenosis. Due to limited data, only preliminary information is obtained regarding potential risk factors affecting the formation of stenosis, and analyses do not include risk factor stratification.

We conclude that the development of esophageal stenosis following ESD is associated with lesion depth and extent.

Acknowledgements We would like to thank each of the authors for their contributions to this study. This research was possible due to support from the following sources: A 2017 grant from the Jiangsu Medical Innovation Team called the Comprehensive Prevention and Treatment of Early Gastric Cancer (CXTDA2017033); the National Natural Science Foundation of China (81770561); the Construction Program of Jiangsu Provincial Clinical Research Center Support System (BL2014084); and the project named "The effectiveness and mechanism of local injection of botulinum toxin A for esophageal stricture after endoscopic submucosal dissection" (2017CX020).

Author contributions MC was the main contributor and the first author of this paper. YD planned and organized the paper; MC and JY extracted the data; $\mathrm{MC}$ analyzed the data; $\mathrm{CD}$ contributed materials/ analysis tools; MC contributed to the writing of the manuscript; GZ and XS proofread the paper.

\section{Compliance with ethical standards}

Disclosures Drs. Meihong Chen, Xinmin Si, Yini Dang, Chao Ding, Jiajia Yang and Guoxin Zhang have no conflicts of interest or financial ties to disclose.

Open Access This article is licensed under a Creative Commons Attribution 4.0 International License, which permits use, sharing, adaptation, distribution and reproduction in any medium or format, as long as you give appropriate credit to the original author(s) and the source, provide a link to the Creative Commons licence, and indicate if changes were made. The images or other third party material in this article are included in the article's Creative Commons licence, unless indicated otherwise in a credit line to the material. If material is not included in the article's Creative Commons licence and your intended use is not permitted by statutory regulation or exceeds the permitted use, you will need to obtain permission directly from the copyright holder. To view a copy of this licence, visit http://creativecommons.org/licenses/by/4.0/.

\section{References}

1. Yang D, Othman M, Draganov PV (2019) Endoscopic mucosal resection vs endoscopic submucosal dissection for barrett's esophagus and colorectal neoplasia. Clin Gastroenterol Hepatol 17(6):1019-1028

2. Guo HM, Zhang XQ, Chen M, Huang SL, Zou XP (2014) Endoscopic submucosal dissection vs endoscopic mucosal 
resection for superficial esophageal cancer. World J Gastroenterol 20(18):5540-5547

3. Nakagawa K, Koike T, Iijima K et al (2014) Comparison of the long-term outcomes of endoscopic resection for superficial squamous cell carcinoma and adenocarcinoma of the esophagus in Japan. Am J Gastroenterol 109(3):348-356

4. Pech O, May A, Manner H et al (2014) Long-term efficacy and safety of endoscopic resection for patients with mucosal adenocarcinoma of the esophagus. Gastroenterology 146(3):652-660

5. Ono S, Fujishiro M, Niimi K et al (2009) Long-term outcomes of endoscopic submucosal dissection for superficial esophageal squamous cell neoplasms. Gastrointest Endosc 70(5):860-866

6. Domper AM, Ferrandez AA, Lanas AA (2015) Esophageal cancer: risk factors, screening and endoscopic treatment in western and eastern countries. World J Gastroenterol 21(26):7933-7943

7. Shaheen NJ, Sharma P, Overholt BF et al (2009) Radiofrequency ablation in barrett's esophagus with dysplasia. N Engl J Med 360(22):2277-2288

8. Chang KJ (2019) Endoscopic foregut surgery and interventions: The future is now. The state-of-the-art and my personal journey. World J Gastroenterol 25(1):1-41

9. Shi Q, Ju H, Yao LQ, Zhou PH, Xu MD, Chen T, Zhou JM, Chen TY, Zhong YS (2014) Risk factors for postoperative stricture after endoscopic submucosal dissection for superficial esophageal carcinoma. Endoscopy 46(8):640-644

10. Funakawa K, Uto H, Sasaki F et al (2015) Effect of endoscopic submucosal dissection for superficial esophageal neoplasms and risk factors for postoperative stricture. Medicine 94(1):e373

11. Katada C, Muto M, Manabe T, Boku N, Ohtsu A, Yoshida S (2003) Esophageal stenosis after endoscopic mucosal resection of superficial esophageal lesions. Gastrointest Endosc 57(2):165-169

12. Lewis JJM, Rubenstein JHMM, Singal AGMM, Elmunzer BJM, Kwon RSM, Piraka CRM (2011) Factors associated with esophageal stricture formation after endoscopic mucosal resection for neoplastic barrett's esophagus. Gastrointest Endosc 74(4):753-760

13. Mizuta H, Nishimori I, Kuratani Y, Higashidani Y, Kohsaki T, Onishi S (2009) Predictive factors for esophageal stenosis after endoscopic submucosal dissection for superficial esophageal cancer. Dis Esophagus 22(7):626-631

14. Qumseya B, Panossian AM, Rizk C et al (2014) Predictors of esophageal stricture formation post endoscopic mucosal resection. Clin Endosc 47(2): 155

15. Ono S, Fujishiro M, Niimi K et al (2009) Predictors of postoperative stricture after esophageal endoscopic submucosal dissection for superficial squamous cell neoplasms. Endoscopy 41(8):661

16. Aadam AA, Abe S (2018) Endoscopic submucosal dissection for superficial esophageal cancer. Dis Esophagus 31(7):doy021

17. Honda K, Akiho H (2012) Endoscopic submucosal dissection for superficial esophageal squamous cell neoplasms. World J Gastrointest Pathophysiol 3(2):44-50

18. Mochizuki Y, Saito Y, Tanaka T et al (2012) Endoscopic submucosal dissection combined with the placement of biodegradable stents for recurrent esophageal cancer after chemoradiotherapy. J Gastrointest Cancer 43(2):324-328
19. Wen J, Lu Z, Liu Q (2014) Prevention and treatment of esophageal stenosis after endoscopic submucosal dissection for early esophageal cancer. Gastroenterol Res Pract 2014:457101

20. Kawaguchi K, Kurumi H, Takeda Y, Yashima K, Isomoto H (2017) Management of strictures after endoscopic submucosal dissection for superficial esophageal cancer. Ann Transl Med 5(8): 184

21. Liao Z, Liao G, Yang X et al (2018) Transplantation of autologous esophageal mucosa to prevent stricture after circumferential endoscopic submucosal dissection of early esophageal cancer (with video). Gastrointest Endosc 88(3):543-546

22. Nishizawa T, Yahagi N (2017) Endoscopic mucosal resection and endoscopic submucosal dissection: technique and new directions. Curr Opin Gastroenterol 33(5):315-319

23. Balmadrid B, Hwang JH (2015) Endoscopic resection of gastric and esophageal cancer. Gastroenterol Rep (Oxf) 3(4):330-338

24. Ezoe Y, Muto M, Horimatsu T et al (2011) Efficacy of preventive endoscopic balloon dilation for esophageal stricture after endoscopic resection. J Clin Gastroenterol 45(3):222-227

25. Wen J, Lu Z, Yang Y et al (2014) Preventing stricture formation by covered esophageal stent placement after endoscopic submucosal dissection for early esophageal cancer. Dig Dis Sci 59(3):658-663

26. Hashimoto S, Kobayashi M, Takeuchi M, Sato Y, Narisawa R, Aoyagi Y (2011) The efficacy of endoscopic triamcinolone injection for the prevention of esophageal stricture after endoscopic submucosal dissection. Gastrointest Endosc 74(6):1389-1393

27. Mizushima T, Ohnishi S, Hosono H et al (2017) Oral administration of conditioned medium obtained from mesenchymal stem cell culture prevents subsequent stricture formation after esophageal submucosal dissection in pigs. Gastrointest Endosc 86(3):542-552

28. Kanai N, Yamato M, Ohki T, Yamamoto M, Okano T (2012) Fabricated autologous epidermal cell sheets for the prevention of esophageal stricture after circumferential ESD in a porcine model. Gastrointest Endosc 76(4):873-881

29. Hochberger J, Koehler P, Wedi E et al (2014) Transplantation of mucosa from stomach to esophagus to prevent stricture after circumferential endoscopic submucosal dissection of early squamous cell. Gastroenterology 146(4):906-909

30. Yamamoto Y, Kikuchi D, Nagami Y, Nonaka K, Tsuji Y, Fujimoto A, Sanomura Y, Tanaka K, Abe S, Zhang S, De Lusong MA, Uedo N (2019) Management of adverse events related to endoscopic resection of upper gastrointestinal neoplasms: Review of the literature and recommendations from experts. Dig Endosc 31(Suppl 1):4-20

31. Iizuka T, Kikuchi D, Hoteya S, Kaise M (2018) Effectiveness of modified oral steroid administration for preventing esophageal stricture after entire circumferential endoscopic submucosal dissection. Dis Esophagus 31(7):dox140

Publisher's Note Springer Nature remains neutral with regard to jurisdictional claims in published maps and institutional affiliations. 